\title{
Curated Single Cell Multimodal Landmark Datasets for R/Bioconductor
}

Kelly B. Eckenrode ${ }^{1,2 *}$, Dario Righelli ${ }^{3 *}$, Marcel Ramos ${ }^{1,2,4 *}$, Ricard Argelaguet ${ }^{5 *}$, Christophe Vanderaa ${ }^{6 \uparrow}$, Ludwig Geistlinger ${ }^{7 \uparrow}$, Aedin Culhane ${ }^{8}$, Laurent Gatto ${ }^{6}$, Vincent Carey ${ }^{9}$, Martin Morgan $^{4}$, Davide Risso ${ }^{3 * *}$, Levi Waldron ${ }^{1,2 * *}$

*shared first authorship

'shared second authorship

${ }^{* *}$ corresponding authors

\section{Author information}

\section{Affiliations}

1. Graduate School of Public Health and Health Policy, City University of New York, NY, NY, United States

Kelly B. Eckenrode* (kelly.eckenrode@sph.cuny.edu), Marcel Ramos** (marcel.

ramos@sph.cuny.edu), Ludwig Geistlinger* (ludwig.geistlinger@sph.cuny.edu), Levi

Waldron*(levi.waldron@sph.cuny.edu)

2. Institute for Implementation Science in Public Health, City University of New York, NY, NY, United States

Kelly B. Eckenrode*, Marcel Ramos ${ }^{* *}$, Ludwig Geistlinger, ${ }^{*}$ Levi Waldron*

3. Department of Statistical Sciences, University of Padova, Padova, Italy.

Dario Righelli (dario.righelli@unipd.it), Davide Risso (davide.risso@unipd.it)

4. Roswell Park Comprehensive Cancer Institute, Buffalo, New York, United States Marcel Ramos ${ }^{\star * *}$, Martin Morgan (martin.morgan@roswellpark.org)

5. European Bioinformatics Institute (EMBL-EBI), Hinxton, Cambridgeshire, CB10 1SD, UK.

Ricard Argelaguet (ricard@ebi.ac.uk)

6. de Duve Institute, Université catholique de Louvain, Avenue Hippocrate 75, Brussels, 1200, Belgium 
Christophe Vanderaa (christophe.vanderaa@uclouvain.be), Laurent Gatto

(laurent.gatto@uclouvain.be)

7. Center for Computational Biomedicine, Harvard Medical School, Boston, Massachusetts, United States Ludwig Geistlinger

(Ludwig_Geistlinger@hms.harvard.edu)

8. School of Medicine, University of Limerick, Limerick, Ireland

Aedin Culhane (aedin@ds.dfci.harvard.edu)

9. Channing Division of Network Medicine, Brigham and Women's Hospital and Harvard Medical School, Boston, Massachusetts, United States

Vincent Carey (stvjc@channing.harvard.edu)

Corresponding authors

Davide Risso, davide.risso@unipd.it

Levi Waldron, levi.waldron@sph.cuny.edu 


\section{Abstract}

Background: The majority of high-throughput single-cell molecular profiling methods quantify RNA expression; however, recent multimodal profiling methods add simultaneous measurement of genomic, proteomic, epigenetic, and/or spatial information on the same cells. The development of new statistical and computational methods in Bioconductor for such data will be facilitated by easy availability of landmark datasets using standard data classes.

Results: We collected, processed, and packaged publicly available landmark datasets from important single-cell multimodal protocols, including CITE-Seq, ECCITE-Seq, SCoPE2, scNMT, 10X Multiome, seqFISH, and G\&T. We integrate data modalities via the MultiAssayExperiment Bioconductor class, document and re-distribute datasets as the SingleCellMultiModal package in Bioconductor's Cloud-based ExperimentHub. The result is single-command actualization of landmark datasets from seven single-cell multimodal data generation technologies, without need for further data processing or wrangling in order to analyze and develop methods within Bioconductor's ecosystem of hundreds of packages for single-cell and multimodal data.

Conclusions: We provide two examples of integrative analyses that are greatly simplified by SingleCellMultiModal. The package will facilitate development of bioinformatic and statistical methods in Bioconductor to meet the challenges of integrating molecular layers and analyzing phenotypic outputs including cell differentiation, activity, and disease.

Keywords: Single cell multimodal, Bioconductor, Data analysis, Genomics, Transcriptomics, Proteomics, spatial transcriptomics, bioinformatics 


\section{Introduction}

Understanding the quantitative relationship between molecules and physiology has motivated the development of quantitative profiling techniques, especially for single-cell sequencing [1]. Single-cell multimodal omics technologies (Nature Method of the Year 2019 [2]) couple single-cell RNA sequencing with other molecular profiles such as DNA sequences, methylation, chromatin accessibility, cell surface proteins, and spatial information, simultaneously in the same cell. Integrative analysis of multiple molecular measurements from the same cell has enabled, for example, discovery of rare cell types by defining subpopulations based on surface markers with CITE-Seq [3] and ECCITE-Seq [4] (Cellular Indexing of Transcriptomes and Epitopes by sequencing, Expanded CRISPR CITE-Seq), of epigenetic regulation and cell differentiation lineage with scNMT-seq [5] (single-cell nucleosome, methylation, and transcriptome sequencing), a high resolution commercial version of single cell chromatin accessibility with 10X Multiomics [20], understanding of spatial patterns of gene expression with seq-FISH [6], and correlation of genotype-phenotype in healthy and disease states with G\&T-seq [7] (parallel Genome and Transcriptome sequencing). Other single-cell multimodal datasets take measurements from separate cells due to the technical constraints, like mass-spectrometry based proteomic methods including SCoPE2 [8] (single-cell protein analysis by mass spectrometry).

Capturing and integrating an array of different molecular signals at the single-cell level poses new analytical challenges. Single-cell multimodal experiments generate multidimensional and high volume datasets, requiring distinct informatic and statistical methods to store, process and analyze data. Integrating different molecular layers to provide biologically meaningful insight is an active area of development in R/Bioconductor due to the availability of data containers and analysis toolkits for single-cell analysis. R/Bioconductor is an open development and open source platform for analyzing biomedical and genomic data with dedicated data structures such as the 
SingleCellExperiment class [9] for single-cell data and the MultiAssayExperiment class [10] for multi-omics data. Both are designed based on the SummarizedExperiment class [11], the central Bioconductor data structure for storing, manipulating, and analyzing high-throughput quantitative omics data. Relative to analysis platforms within and outside of the R programming language (e.g. GATK, Seurat [12], mixOmics [13], MOFA+ [14], CiteFuse package [15], ScanPy for CITE-Seq [16], Conos for SCoPE2 [17]), Bioconductor provides the broadest range of interoperable data structures and packages for statistical analysis and visualization of single-cell multimodal data.

Easy availability of publicly available experimental data using standardized data classes has long played an important role in the development of interoperable software packages for the analysis of data from new technologies, helping to coalesce development efforts around shared datasets and commonly used data classes such as ExpressionSet [18] and then (Ranged)SummarizedExperiment [19] and SingleCellExperiment [9]. We therefore introduce a suite of single-cell multimodal landmark datasets for benchmarking and testing multimodal analysis methods via the Bioconductor ExperimentHub package SingleCellMultiModal (Figure 1A). The scope of this package is to provide efficient access to a selection of curated, preintegrated, publicly available landmark datasets for methods development and benchmarking within the Bioconductor ecosystem. Some such methods and code for analysis workflows are reviewed by Lê Cao et al. [20]. Users can obtain integrative representations of multiple modalities as a MultiAssayExperiment, a common core Bioconductor data structure relied on by dozens of multimodal data analysis packages . SingleCellMultiModal uses Bioconductor's ExperimentHub package and service to host, coordinate, and manage the data from the cloud. We plan to update the package as new datasets and technologies become available and we welcome community contributions. This manuscript serves as a review of essential aspects of these technologies suitable for developers of bioinformatic and statistical software, and as a description of the SingleCellMultiModal data package. 


\section{Summary of landmark datasets in SingleCellMultiModal}

To evaluate and design new statistical methods that accompany experimental single-cell multimodal data, it is important to establish landmark datasets. The goal of this section is to provide an overview of the landmark datasets currently in SingleCellMultiModal as well as to introduce the experimental and technological context for each experimental assay (Table 1). For more information concerning the details of the technologies, consult [21]. We briefly describe each landmark experiment including context, major findings from the publication, and challenges in its analysis, then summarize its accompanying dataset in SingleCellMultiModal including number of cells and features (Figure 1B). 


\begin{tabular}{|c|c|c|c|c|}
\hline MODALITIES & $\begin{array}{l}\text { EXPERIMENTAL } \\
\text { ASSAY }\end{array}$ & $\begin{array}{l}\text { CELL / } \\
\text { PROCESS } \\
\text { TYPE } \\
\end{array}$ & DATATYPE NAME & CITATION \\
\hline RNA + DNA & G\&T-seq & $\begin{array}{l}\text { Mouse } \\
\text { epithelial, } \\
\text { human breast } \\
\text { tumor }\end{array}$ & mouse_embryo_8_cell & [7] \\
\hline \multirow[t]{2}{*}{ RNA + Protein } & CITE-Seq & $\begin{array}{l}\text { Cord blood } \\
\text { mononuclear }\end{array}$ & cord_blood & [3] \\
\hline & ECCITE-Seq & $\begin{array}{l}\text { Peripheral blood } \\
\text { mononuclear, } \\
\text { human T-cell } \\
\text { lymphoma, } \\
\text { mouse fibroblast }\end{array}$ & peripheral_blood & {$[4]$} \\
\hline \multirow[t]{2}{*}{$\begin{array}{l}\text { RNA + } \\
\text { Epigenetic }\end{array}$} & scNMT-seq & $\begin{array}{l}\text { Mouse } \\
\text { gastrulation }\end{array}$ & mouse_gastrulation & {$[22]$} \\
\hline & $\begin{array}{l}\text { 10X Multiome } \\
\text { scATAC-seq + } \\
\text { Single-cell RNA- } \\
\text { seq }\end{array}$ & $\begin{array}{l}\text { Peripheral blood } \\
\text { mononuclear }\end{array}$ & pbmc_10x & {$[23]$} \\
\hline RNA + Spatial & seqFISH & $\begin{array}{l}\text { Mouse cortical } \\
\text { neuronal }\end{array}$ & mouse_visual_cortex & {$[24],[21]$} \\
\hline $\begin{array}{l}\text { RNA + } \\
\text { Proteomic }\end{array}$ & SCOPE2 & $\begin{array}{l}\text { Human } \\
\text { monocyte and } \\
\text { PMA-induced } \\
\text { macrophage }\end{array}$ & macrophage_differentiation & {$[8]$} \\
\hline
\end{tabular}

Table 1: Single-cell multimodal datasets included SingleCellMultiModal package. Modalities refer to the molecular feature measured in the experimental assay. Cell/process type provides information on the type of material or development event data was collected. Datatype name column refers to the dataset name in SingleCellMultiModal 
A

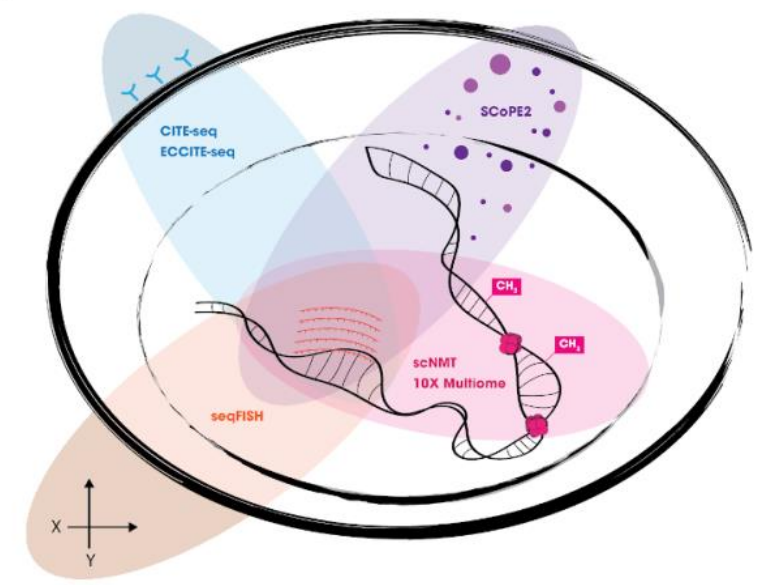

B

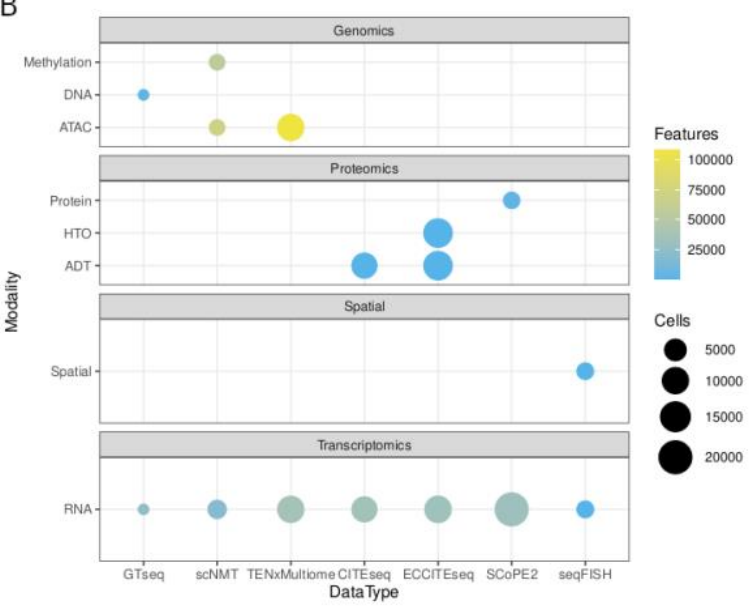

Figure 1: Representation of modalities included in the SingleCellMultiModal package. (A) a Venn diagram representation of the modalities collected by each different technology, including: RNA (center), surface proteins (top left), spatial information (bottom left), methylation and open chromatin (bottom right), and peptides (top right). (B) The number of features and cells collected for each data modality by each technology.

\section{RNA and Protein: antibody tagged cell surface markers}

Purpose: Traditionally, protein expression in cell populations are measured using flow cytometry. With the advent of single-cell multimodal methods cell surface proteins are measured with higher resolution with simultaneous measurements of mRNA abundance, which enhances the ability to identify new cell subpopulations in heterogeneous samples. Cellular Indexing of Transcriptomes and Epitopes by sequencing (CITE-Seq) measures protein cell surface markers and gene expression in the same cell. An extension of CITE-Seq is ECCITE-Seq, Expanded CRISPRcompatible CITE-Seq, which allows for the capture of sgRNA from CRISPR mediated screens. Collectively, these technologies provide a high-throughput method for single-cell immunophenotyping and transcriptome analysis.

Technology: CITE-Seq relies on antibodies conjugated to DNA barcodes to infer protein levels, and in tandem count DNA handles from PCR amplification of mRNA transcripts. Inside the droplet contains mRNA transcripts, proteins conjugated with antibody derived tags (ADTs), beads 
decorated with oligo-dT, reverse transcriptase and primers for cDNA amplification. The use of DNA barcodes is a departure from traditional fluorescence labels, which are limited in number because of the overlaps in spectral detection, excitation and emission frequencies [25].

A variation of CITE-Seq is ECCITE-Seq that can track single-cell CRISPR screens using sgRNA sequencing capture [4]. The CRISPR-Cas9 system is used to generate targeted gene knockout/mutants by using two components: sgRNA (single guide RNA for gene of interest) and Cas9 (endonuclease for cleaving double DNA strand breaks). sgRNA are composed of custom crRNA 17-22nt with a scaffold tracrRNA, which means the sgRNA are composed of two RNA pieces: one is customizable and the other is not. The sgRNA targets the gene of interest and orchestrates the Cas9 enzyme to gene location to insert a variety of mutations or full gene knockouts. The CRISPR-Cas9 system introduces targeted gene mutations with greater ease at the bench, plus it is easier to scale up to many more experimental samples than previous approaches.

Antibody oligo counts are listed in the ADT and HTO (hashtag oligo) tables and sgRNAs counts in the GDO tables. After cell perturbations via CRISPR screens, cells are collected and prepared with 10X Genomics V(D)J solution which incorporates Single-cell RNA-seq with additional profiling of protein surface markers and sgRNAs (when applicable). The molecular contents, mRNA and DNA-tagged proteins, will hybridize to the decorated beads. The benefit of adding barcoding to cells is that it allows for tracking of doublets (two cells in one droplet).

Landmark data: There are several experimental datasets derived from the original CITE-Seq landmark paper. Among them we selected the cord blood dataset where the cells have been incubated with CITE-seq antibody conjugates and fluorophore-conjugated antibodies. This cord_blood dataset has two different assays. The scADT assay is a matrix indicating the 13 proteins surface abundance for each of the 8617 cells, while the scRNA assay is a matrix of 20400 human genes and 15880 mouse genes where each entry contains the expression abundance in each of the 8617 cells (Table 2). 


\begin{tabular}{|l|l|l|l|l|l|l|l|}
\hline $\begin{array}{l}\text { Dataset } \\
\text { Identifier }\end{array}$ & $\begin{array}{l}\text { Assay } \\
\text { Type }\end{array}$ & Modes & Species & $\begin{array}{l}\text { Data } \\
\text { Structure }\end{array}$ & Version & \# features & \# cells \\
\hline Cord blood & RNA-seq & Transcripts & Human & matrix & 1.0 .0 & 36280 & 8617 \\
\cline { 2 - 7 } & ADT & Proteins & Human & matrix & 1.0 .0 & 13 & 8617 \\
\hline
\end{tabular}

Table 2: CITE-Seq dataset description, with assay types, molecular modes, number of specimens, number of features and number of cells. ADTs, antibody derived tags

The package also includes an ECCITE-Seq dataset aimed at characterizing immune subpopulation cell types after an experimental perturbation. The peripheral_blood dataset is organized in two different conditions: the control (CTRL) and the cutaneous T-cell lymphoma (CTCL). For both conditions the ECCITE-Seq protocol has been performed to produce transcripts (RNA-seq), proteins (ADT) and cell tracking (HTO) abundance. All these modalities are collected as separated assays into the MultiAssayExperiment, where a sparse matrix is used to store the RNA-seq counts. The modalities are collected from the same cells, but not all the cells are entirely profiled by the same modalities. Of the total 36248 cells, 4190 cells from the CTCL and 4292 cells from the CTRL are matched with all modalities (Figure 2). sgRNA data is stored in long format providing access through the metadata data structure of the MultiAssayExperiment. The CITESeq dataset is accessible via the SingleCellMultiModal package by using the CITEseq (DataType="cord_blood") function call, while for the ECCITE-Seq data it's sufficient to change the identifier as follow CITEseq(DataType="peripheral_blood"). Both function calls return a MultiAssayExperiment object with matrices or sparse matrices as assays (Table 3). 


\begin{tabular}{|c|c|c|c|c|c|c|c|}
\hline $\begin{array}{l}\text { Dataset } \\
\text { Identifier }\end{array}$ & $\begin{array}{l}\text { Assay } \\
\text { Type }\end{array}$ & Modes & Species & $\begin{array}{l}\text { Data } \\
\text { Structure }\end{array}$ & Condition & \# features & \# cells \\
\hline \multirow{11}{*}{$\begin{array}{l}\text { Peripheral } \\
\text { blood }\end{array}$} & \multirow[t]{2}{*}{ RNA-seq } & \multirow[t]{2}{*}{ Transcripts } & \multirow[t]{2}{*}{ Human } & \multirow[t]{2}{*}{ dgCMatrix } & CTCL & 33538 & 5399 \\
\hline & & & & & CTRL & 33538 & 4849 \\
\hline & \multirow[t]{2}{*}{ ADT } & \multirow[t]{2}{*}{ Proteins } & \multirow[t]{2}{*}{ Human } & \multirow[t]{2}{*}{ dgCMatrix } & CTCL & 52 & 6500 \\
\hline & & & & & CTRL & 52 & 6500 \\
\hline & \multirow[t]{2}{*}{ HTO } & \multirow[t]{2}{*}{ Cell tracking } & \multirow[t]{2}{*}{ Human } & \multirow[t]{2}{*}{ dgCMatrix } & CTCL & 7 & 6500 \\
\hline & & & & & CTRL & 7 & 6500 \\
\hline & \multicolumn{5}{|c|}{ sgRNAs stored in long format } & \# rows & \# cols \\
\hline & \multirow[t]{4}{*}{ sgRNAs } & \multirow[t]{4}{*}{$\begin{array}{l}\text { CRISPR } \\
\text { perturbation }\end{array}$} & \multirow[t]{4}{*}{ Human } & \multirow[t]{4}{*}{ data.frame } & $\begin{array}{l}\text { CTCL } \\
\text { TCRab }\end{array}$ & 9626 & 18 \\
\hline & & & & & $\begin{array}{l}\text { CTCL } \\
\text { TCRgd }\end{array}$ & 2430 & 18 \\
\hline & & & & & $\begin{array}{l}\text { CTRL } \\
\text { TCRab }\end{array}$ & 8359 & 18 \\
\hline & & & & & $\begin{array}{l}\text { CTRL } \\
\text { TCRgd }\end{array}$ & 3099 & 18 \\
\hline
\end{tabular}

Table 3: ECCITE-Seq dataset description: assay types, molecular modes, number of specimens, number of features and number of cells. ADTs, antibody derived tags; HTO, Hashtagged oligos; sgRNAs, CRISPR V(D)J's. 


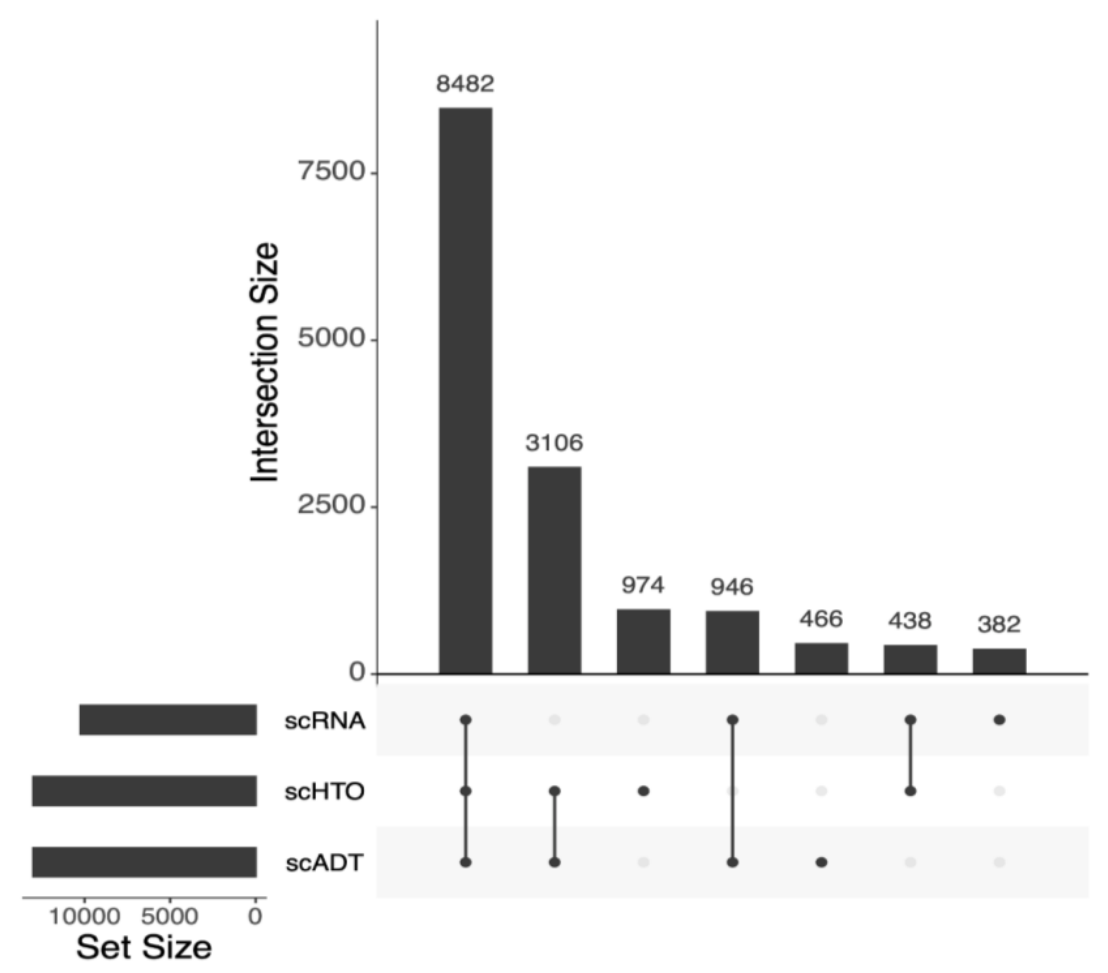

Figure 2: Upset plot [26] of the overlap of modalities on the same cells in the control sample of the ECCITE-Seq "peripheral blood" dataset. 8482 cells are assayed in all three modes (ADT, HTO, RNA), 3105 cells are assayed by HTO and ADT only, etc. RNA data are available for 10248 cells, whereas HTO and ADT data are each individually available for $\sim 13000$ cells across both conditions. This plot is produced by the upsetSamples function of the MultiAssayExperiment package, and can be applied directly to all datasets produced by SingleCellMultiModal.

\section{RNA and Protein: mass spectrometry-based}

Purpose and goals: CITE-Seq offers valuable information about the expression of surface proteins. However, the acquisition is limited to tens of targets as the identification relies on antibodies. Furthermore, it cannot provide information on intracellular markers. Mass spectrometry (MS)-based single-cell proteomics (SCP) provides a means to overcome these limitations and to perform unbiased single-cell profiling of the soluble proteome. MS-SCP is 
emerging thanks to recent advances in sample preparation, liquid chromatography (LC) and MS acquisition. The technology is in its infancy and protocols still need to be adapted in order to acquire multiple multimodalities from a single-cell. In this section the multimodality is achieved by subjecting similar samples to MS-SCP and Single-cell RNA-seq.

Technology: The current state-of-the-art protocol for performing MS-SCP is the SCoPE2 protocol [8]. Briefly, single-cells are lysed, proteins are extracted and digested into peptides. The peptides are then labeled using tandem mass tags (TMT) in order to multiplex up to 16 samples per run (Figure 3A). The pooled peptides are then analysed by LC-MS/MS. LC separates the peptides based on their mass and affinity for the chromatographic column. The peptides are immediately ionized as they come out (Figure 3B) and are sent for two rounds of MS (MS/MS, Figure 3C). The first round isolates the ions based on their mass to charge $(\mathrm{m} / \mathrm{z})$ value. The isolated ions are fragmented and sent to the second round of MS that records the $\mathrm{m} / \mathrm{z}$ and intensity of each fragment. The pattern of intensities over $\mathrm{m} / \mathrm{z}$ value generated by an ion is called an MS2 spectrum. The MS2 spectra are then computationally matched to a database to identify the original peptide sequence from which they originated. The spectra that were successfully associated to a peptide sequence are called peptide to spectrum matches (PSMs, Figure 3D). Next to that, a specific range of the MS spectrum holds the TMT label information where each label generates a fragment with an expected $\mathrm{m} / \mathrm{z}$ value. The intensity of each label peak is proportional to the peptide expression in the corresponding single-cell and this allows for peptide quantification (Figure 3D). Finally, the quantified PSM data go through a data processing pipeline that aims to reconstruct the protein data that can be used for downstream analyses (Figure 3E). 


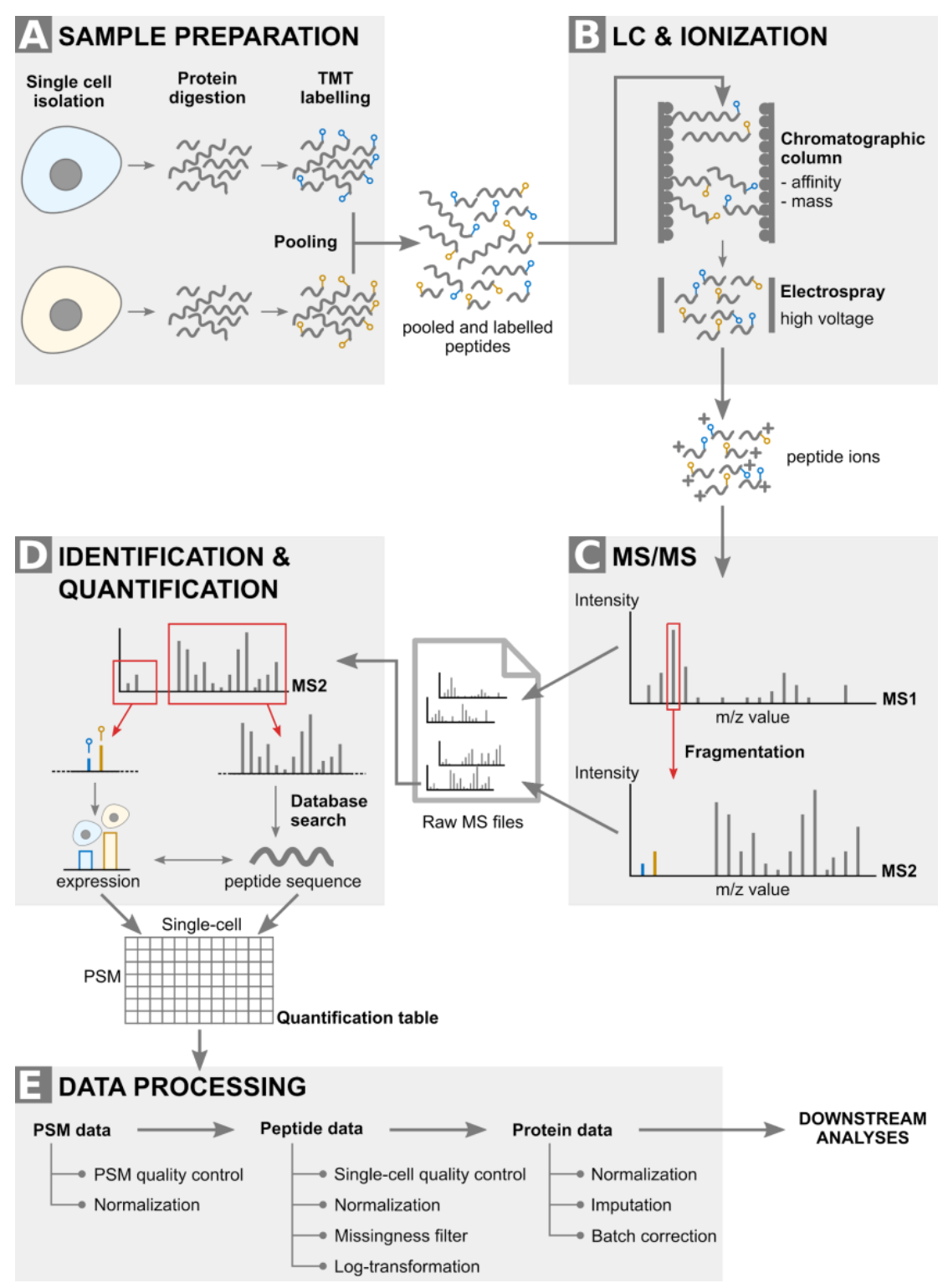

Figure 3: SCoPE2 workflow. The workflow consists of 4 main steps. (A) Sample preparation extracts and labels peptides from single-cells. (B) LC separates the peptides based on their mass and affinity for the column. Note that the TMT tag does not influence those properties. Peptides that are eluting are ionised thanks to an electrospray. (C) MS/MS performs an $\mathrm{m} / \mathrm{z}$ scan of the incoming ions to select the most abundant ones that are then fragmented separately. A second round of MS acquires the spectrum generated by the ion fragments. (D) Each spectrum is then computationally processed to obtain the cell-specific expression values and the peptide identity. (E) The data processing pipeline reconstructs the protein data from the quantified PSMs. Abbreviations: TMT: tandem mass tags; LC: liquid chromatography; MS: mass spectrometry; MS/MS: tandem MS; m/z: mass over charge; PSM: peptide to spectrum match. 
The major challenge in MS-SCP is to recover sufficient peptide material for accurate peptide identification and quantification. SCoPE2 solves this issue by optimizing the sample preparation step to limit samples loss, by providing analytical tools to optimize the MS/MS settings, and most importantly by introducing a carrier sample into the pool of multiplexed samples. The carrier is a sample that contains hundreds of cells instead of a single-cell and allows to boost the peptide identification rate by increasing the amount of peptide material delivered to the MS instrument.

Parallel to SCoPE2, other groups have developed a label-free MS-SCP, where each LCMS/MS run contains unlabelled peptides from a single cell [27]. Although it allows for more accurate quantifications, it suffers from low throughput. The current methodological advances in MS-SCP have extensively been reviewed elsewhere [28].

Landmark data: The SCoPE2 dataset we provide in this work was retrieved from the supplementary information of the landmark paper [8]. This is a milestone dataset as it is the first publication where over a thousand cells are measured by MS-SCP. The research question is to understand whether a homogeneous monocyte population ( $U-937$ cell line) could differentiate upon PMA treatment into a heterogeneous macrophage population, namely whether M1 and M2 macrophage profiles could be retrieved in the absence of differentiation cytokines. Different replicates of monocyte and macrophage samples were prepared and analyzed using either MSSCP or Single-cell RNA-seq. The MS-SCP data was acquired in 177 batches with on average 9 single-cells per batch. The Single-cell RNA-seq data was acquired in 2 replicates with on average 10,000 single-cells per acquisition using the 10x Genomics Chromium platform. Cell type annotations are only available for the MS-SCP data. Note also that MS-SCP data provides expression information at protein level meaning that the peptide data has already been processed. The processing includes filtering high quality features, filtering high quality cells, logtransformation, normalization, aggregation from peptides to proteins, imputation and batch correction (Figure 3E). More details on the protein data processing can be found in the original 
paper or in the paper that reproduced that analysis [29]. Count tables were provided for the Singlecell RNA-seq dataset with no additional processing.

The data can be accessed in the SingleCellMultiModal package by calling SCoPE2("macrophage_differentiation") (Table 4). Relevant cell metadata is provided within the MultiAssayExperiment object. The MS-SCP dataset contains expression values for 3,042 proteins in 1,490 cells. The Single-cell RNA-seq contains expression values for 32,738 genes (out of which 10,149 are zero) for 20,274 cells.

\begin{tabular}{|l|l|l|l|l|l|l|l|}
\hline $\begin{array}{l}\text { Dataset } \\
\text { Identifier }\end{array}$ & $\begin{array}{l}\text { Assay } \\
\text { Type }\end{array}$ & Modes & Species & $\begin{array}{l}\text { Data } \\
\text { Structure }\end{array}$ & Version & $\begin{array}{l}\# \\
\text { features }\end{array}$ & \# cells \\
\hline $\begin{array}{l}\text { macrophage } \\
\text { differentiation }\end{array}$ & LC-MS/MS & Proteins & Human & matrix & 1.0 .0 & 3,042 & 1,490 \\
\cline { 2 - 8 } & RNA-seq & Transcripts & Human & HDF5 & 1.0 .0 & $32,738^{1}$ & 20,274 \\
\hline
\end{tabular}

Table 4: SCoPE2 dataset descriptions, with assay types, molecular modes, specimens, dataset version provided, number of features and number of cells

\section{Single-cell nucleosome, methylation and transcription sequencing (scNMT-seq)}

Purpose: The profiling of the epigenome at single-cell resolution has received increasing interest, as it provides valuable insights into the regulatory landscape of the genome [30,31]. Although the term epigenome comprises multiple molecular layers, the profiling of chromatin accessibility and DNA methylation have received the most attention to date.

Technology: DNA methylation is generally measured using single-cell bisulfite sequencing (scBS-seq) [32]. The underlying principle of scBS-seq is the treatment of the DNA with sodium bisulfite before DNA sequencing, which converts unmethylated cytosine $(C)$ residues to uracil (and after retro-PCR amplification, to thymine (T)), leaving 5-methylcytosine residues intact. The resulting $\mathrm{C} \rightarrow \mathrm{T}$ transitions can then be detected by DNA sequencing. Further methodological

${ }^{1} 10,149$ out of the 32,738 features are zero 
innovations enabled DNA methylation and RNA expression to be profiled from the same cell, demonstrated by the scM\&T-seq assay [33].

Chromatin accessibility was traditionally profiled in bulk samples using DNase sequencing (DNase-seq) [34]. However, in recent years, transposase-accessible chromatin followed by sequencing (ATAC-seq) has displaced DNase-seq as the de facto method for profiling chromatin accessibility due to its fast and sensitive protocol, most notably in single-cell genomics [35]. Briefly, in ATAC-seq, cells are incubated with a hyperactive mutant Tn5 transposase, an enzyme that inserts artificial sequencing adapters into nucleosome-free regions. Subsequently, the adaptors are purified, PCR-amplified and sequenced. Notably, single-cell ATAC-seq has also been combined with Single-cell RNA-seq to simultaneously survey RNA expression and chromatin accessibility from the same cell, as demonstrated by SNARE-seq [36], SHARE-seq [37] and the recently commercialised Multiome Kit from 10x Genomics [23]. Finally, some assays have been devised to capture at least three molecular layers from the same cell, albeit at a lower throughput than SNARE-seq or SHARE-seq. An example is scNMT-seq (single-cell nucleosome methylation and transcriptome sequencing) [5]. scNMT captures a snapshot of RNA expression, DNA methylation and chromatin accessibility in single-cells by combining two previous multimodal protocols: scM\&T-seq [33] and Nucleosome Occupancy and Methylation sequencing (NOMe-seq) [38]

In the first step (the NOMe-seq step), cells are sorted into individual wells and incubated with a GpC methyltransferase. This enzyme labels accessible (or nucleosome depleted) GpC sites via DNA methylation. In mammalian genomes, cytosine residues in GpC dinucleotides are methylated at a very low rate. Hence, after the GpC methyltransferase treatment, GpC methylation marks can be interpreted as direct readouts for chromatin accessibility, as opposed to the CpG methylation readouts, which can be interpreted as endogenous DNA methylation. In a second step (the scM\&T-seq step), the DNA molecules are separated from the mRNA using 
oligo-dT probes pre-annealed to magnetic beads. Subsequently, the DNA fraction undergoes scBS, whereas the RNA fraction undergoes Single-cell RNA-seq.

Landmark data: The scNMT landmark paper reported simultaneous measurements of chromatin accessibility, DNA methylation, and RNA expression at single-cell resolution during early embryonic development, spanning exit from pluripotency to primary germ layer specification [22]. This dataset represents the first multi-omics roadmap of mouse gastrulation at single-cell resolution. Using multi-omic integration methods, the authors detected genomic associations between distal regulatory regions and transcription activity, revealing novel insights into the role of the epigenome in regulating this key developmental process.

One of the challenges of this dataset is the complex missing value structure. Whereas RNA expression is profiled for most cells $(\mathrm{N}=2480)$, DNA methylation and chromatin accessibility is only profiled for subsets of cells ( $\mathrm{N}=986$ and $\mathrm{N}=1105$, respectively). This poses important challenges to some of the conventional statistical methods that do not handle missing information.

The output of the epigenetic layers from scNMT-seq is a binary methylation state for each observed CpG (endogenous DNA methylation) and GpC (a proxy for chromatin accessibility). However, instead of working at the single nucleotide level, epigenetic measurements are typically quantified over genomic features (i.e. promoters, enhancers, etc.). This is done assuming a binomial model for each cell and feature, where the number of successes is the number of methylated $\mathrm{CpGs}$ (or GpCs) and the number of trials is the total number of CpGs (or GpCs) that are observed. Here we provide DNA methylation and chromatin accessibility estimates quantified over CpG islands, gene promoters, gene bodies and DNAse hypersensitive sites (defined in Embryonic Stem Cells).

The pre-integrated scNMT dataset is accessed from the SingleCellMultiModal package by calling e.g. scNMT("mouse_gastrulation", version $=" 1.0 .0 "$ ) (Table 5). Relevant cell metadata is provided within the MultiAssayExperiment object. The overall dataset is $277 \mathrm{MB}$. 


\begin{tabular}{|c|c|c|c|c|c|}
\hline $\begin{array}{l}\text { Dataset } \\
\text { Identifier }\end{array}$ & Assay type & Modes & $\begin{array}{l}\text { Data } \\
\text { structure }\end{array}$ & \# features & \# cells \\
\hline \multirow{9}{*}{$\begin{array}{l}\text { Mouse } \\
\text { Gastrulation }\end{array}$} & RNA-seq & Transcripts & Matrix & 18345 & 2480 \\
\hline & \multirow[t]{4}{*}{$\begin{array}{l}\text { DNA } \\
\text { Methylation }\end{array}$} & CpG islands & Matrix & 14080 & 986 \\
\hline & & promoters & Matrix & 17179 & 986 \\
\hline & & Gene bodies & Matrix & 17559 & 986 \\
\hline & & DHS & Matrix & 6673 & 986 \\
\hline & \multirow[t]{4}{*}{$\begin{array}{l}\text { Chromatin } \\
\text { accessibility }\end{array}$} & CpG islands & Matrix & 14824 & 1101 \\
\hline & & promoters & Matrix & 18037 & 1103 \\
\hline & & Gene bodies & Matrix & 17924 & 1105 \\
\hline & & DHS & Matrix & 20082 & 1094 \\
\hline
\end{tabular}

Table 5: scNMT-seq dataset description, with of assay types, molecular modes, number of specimens, number of features and number of cells

\section{Chromium Single-cell Multiome ATAC and gene expression}

Purpose: A new commercial platform introduced in late 2020 by $10 X$ Genomics, the Chromium Single Cell Multiome ATAC and gene expression (10x Multiome), provides simultaneous gene expression and open chromatin measurements from the same cell at high throughput. This technology is well suited to identify gene regulatory networks by linking open chromatin regions with changes in gene expression, a task which is harder to perform when the two modalities are derived from separate groups of cells. However, very few datasets have been published to date using the 10x Multiome technology, and so how much information can be obtained by simultaneously profiling both modalities in the same cell remains an open question. 
Technology: First, cells are purified and single nuclei are isolated, chromosomes are transpositioned. Next, ATAC and mRNA sequencing libraries are prepared with 10X Genomics Chromium microfluidic controller device where nuclei are partitioned and embedded in a droplet with a decorated gel bead with DNA 16nt 10X barcode that allows for pairing ATAC and mRNA signals to the same nuclei. mRNA is tagged with an 12nt Unique Molecular Identifier sequence (UMI), and a poly(dT)VN for poly-adenylated 3'ends. ATAC fragments are tagged with a Illumina primer sequence and an 8nt space sequence. All barcoded products are amplified in two rounds of PCR and then processed for sequencing. According to the Chromium Single-Cell Multiome ATAC and gene expression assay product information, it has a flexible throughput of $500-10,000$ nuclei per channel and up to 80,000 per run with a $65 \%$ recovery rate and low multiplet rate of $<1 \%$ per 1000 cells (10Xgenomics.com).

Landmark data: 10X genomics has released a dataset of $\sim 10 \mathrm{k}$ peripheral blood mononuclear cells (PBMCs) from a human healthy donor. Here we provide the RNA expression matrix and the binary matrix of ATAC fragments for each cell, quantified over a set of pre-computed peaks (Table 6). To access data in the SingleCellMultiModal package, call the scMultiome ("pbmc_10x") command. Relevant cell metadata is provided within the MultiAssayExperiment object. The overall dataset is $1.1 \mathrm{~GB}$.

\begin{tabular}{|l|l|l|l|l|l|}
\hline $\begin{array}{l}\text { Dataset } \\
\text { Identifier }\end{array}$ & Assay type & Modes & Data structure & \# features & \# cells \\
\hline $\begin{array}{l}\text { Human } \\
\text { PBMCs }\end{array}$ & RNA-seq & $\begin{array}{l}\text { Gene } \\
\text { expression }\end{array}$ & SingleCellExperiment & 36,549 & 10,032 \\
\cline { 2 - 6 } & $\begin{array}{l}\text { Chromatin } \\
\text { accessibility }\end{array}$ & $\begin{array}{l}\text { Fragments } \\
\text { over peaks }\end{array}$ & SingleCellExperiment & 108,344 & 10,032 \\
\hline
\end{tabular}

Table 6: 10X Multiome dataset descriptions, with assay types, molecular modes, number of features and number of cells 


\section{RNA and Spatial sequencing assays}

Purpose and goals: The power of microscopy to resolve spatial information has been paired with single-cell sequencing to measure transcriptomic activity. These microscopy-based sequencing technologies capture a cell population's heterogeneous gene expression typically lost in bulk assays. Technologies like seqFISH(+) (sequential Fluorescence In Situ Hybridization), fluorescence in situ hybridization sequencing [6], Multiplexed error-robust fluorescence in situ hybridization (MERFISH) [39], Slide-seq [40,41] combine sequential barcoding with in situ molecular fluorescence probing, allowing the identification from tens to thousands of mRNAs transcripts while preserving spatial coordinates at micrometer resolution. We refer to this family of technologies as molecular-based spatial transcriptomics. Another family of spatial omics technologies can be described as spot-based; it includes the 10x Visium Spatial Gene Expression and Slide-seq [40]. In this family, the spatial coordinates are typically associated with barcoded spot-like identities, where the transcripts are amplified and sequenced. Currently, our package does not include any spot-based spatial transcriptomics dataset. The TENxVisiumData package [42] (available at https://github.com/HelenaLC/TENxVisiumData) contains several such datasets. See [43] for a comprehensive review of spatial transcriptomics technologies.

Technology: The seqFISH technology makes use of temporal barcodes to be read in multiple rounds of hybridization where mRNAs are labeled with fluorescent probes. During the hybridization rounds, the fluorescent probes are hybridized with the transcripts to be imaged with microscopy. Then they are stripped to be re-used and coupled with different fluorophores, during further rounds. In this case, the transcript abundance is given by the number of colocalizing spots per each transcript. The main differences between the technologies are due to the barcoding of RNAs. In seqFISH they are detected as a color sequence while in MERFISH the barcodes are identified as binary strings allowing error handling but requiring longer transcripts and more rounds of hybridizations [44]. 
Landmark data: The provided seqFISH dataset is designed on a mouse visual cortex tissue and can be retrieved in two different versions. Both versions include Single-cell RNA-seq and seqFISH data. Single-cell RNA-seq data in version 1.0.0 are part of the original paper [24] of 24057 genes in 1809 cells, while version 2.0.0 is a pre-processed adaptation of version 1.0.0 [21] where the authors analyzed it in order to provide the 113 genes in common with seqFISH data in 1723 cells. The provided seqFISH data are the same for both versions as part of their original paper $[45,46]$ made of 1597 cells and 113 genes. The dataset is accessible via the SingleCellMultiModal Bioconductor package by using the seqFISH(DataType="mouse_visual_cortex", version = "1.0.0" ) function call, which returns a MultiAssayExperiment object with a SpatialExperiment object for the seqFISH data and a SingleCellExperiment object for the Single-cell RNA-seq data (Table 7).

\begin{tabular}{|l|l|l|l|l|l|l|l|}
\hline $\begin{array}{l}\text { Dataset } \\
\text { Identifier }\end{array}$ & $\begin{array}{l}\text { Assay } \\
\text { Type }\end{array}$ & Modes & Species & Data Structure & $\begin{array}{l}\text { Versio } \\
n\end{array}$ & $\begin{array}{l}\# \\
\text { features }\end{array}$ & \# cells \\
\hline $\begin{array}{l}\text { Mouse } \\
\text { visual } \\
\text { cortex }\end{array}$ & $\begin{array}{l}\text { Single-cell } \\
\text { RNA-seq }\end{array}$ & Transcripts & Mouse & $\begin{array}{l}\text { SingleCellExpe } \\
\text { riment }\end{array}$ & 1.0 .0 & 24057 & 1809 \\
\cline { 2 - 8 } & seqFISH & $\begin{array}{l}\text { Spatial } \\
\text { Transcripto } \\
\text { mics }\end{array}$ & Mouse & $\begin{array}{l}\text { SpatialExperim } \\
\text { ent }\end{array}$ & $\begin{array}{l}1.0 .0 / \\
2.0 .0\end{array}$ & 113 & 1597 \\
\hline
\end{tabular}

Table 7: seqFISH dataset descriptions, with assay types, molecular modes, specimens, dataset version provided, number of features and number of cells

\section{RNA and DNA sequencing assays}

Purpose and goals: Parallel genome and transcriptome sequencing (G\&T-seq) of single-cells [7] opens new avenues for measuring transcriptional responses to genetic and genomic variation resulting from different allele frequencies, genetic mosaicism [47], single nucleotide variants (SNVs), DNA copy-number variants (CNVs), and structural variants (SVs). Although current experimental protocols are low-throughput with respect to the number of cells, simultaneous DNA 
and RNA sequencing of single-cells resolves the problem of how to associate cells across each modality from independently sampled single-cell measurements [48].

Technology: Following cell isolation and lysis, G\&T-seq measures DNA and RNA levels of the same cell by physically separating polyadenylated RNA from genomic DNA using a biotinylated oligo-dT primer [49]. This is followed by separate whole-genome and whole-transcriptome amplification. Whole-genome amplification is carried out via multiple displacement amplification (MDA) or displacement pre-amplification and PCR (DA-PCR) for DNA sequencing, providing targeted sequencing reads or genome-wide copy number estimates. Parallel Smart-seq2 wholetranscriptome amplification is used for Illumina or PacBio cDNA sequencing, providing gene expression levels based on standard computational RNA-seq quantification pipelines. While pioneering technologies such as G\&T-seq [7] and DR-seq [50] sequence both the DNA and RNA from single-cells, they currently measure only few cells (50-200 cells [51]) compared to assays that sequence DNA or RNA alone (1,000 - 10,000 cells [51]) such as Direct Library Preparation [52] or 10x Genomics Single-cell RNA-seq [53].

Landmark data: G\&T-seq has been applied by Macaulay et al. [7] for parallel analysis of genomes and transcriptomes of (i) 130 individual cells from breast cancer line HCC38 and B lymphoblastoid line HCC38-BL, and (ii) 112 single cells from a mouse embryo at the eight-cell stage. Publicly available and included in the SingleCellMultiModal package is the mouse embryo dataset, assaying blastomeres of seven eight-cell cleavage-stage mouse embryos, five of which were treated with reversine at the four-cell stage of in vitro culture to induce chromosome missegregation. The dataset is stored as a MultiAssayExperiment [10] consisting of (i) a SinglecellExperiment [9] storing the single-cell RNA-seq read counts, and (ii) a RaggedExperiment [54] storing integer copy numbers as previously described [55] (Table 8). Although assaying only a relatively small number of cells, the dataset can serve as a prototype for benchmarking single-cell eQTL integration of DNA copy number and gene expression levels, 
given that Macaulay et al. [7] reported copy gains or losses with concomitant increases and decreases in gene expression levels.

\begin{tabular}{|l|l|l|l|l|l|l|l|}
\hline $\begin{array}{l}\text { Dataset } \\
\text { Identifier }\end{array}$ & $\begin{array}{l}\text { Assay } \\
\text { Type }\end{array}$ & Mode & Species & $\begin{array}{l}\text { Data } \\
\text { Structure }\end{array}$ & Version & \# features & \# cells \\
\hline E-ERAD-381 & RNA-seq & $\begin{array}{l}\text { mRNA } \\
\text { expression }\end{array}$ & Mouse & $\begin{array}{l}\text { SingleCell } \\
\text { Experime } \\
\text { nt }\end{array}$ & 1.0 .0 & 23363 & 112 \\
\cline { 2 - 7 } & DNA-seq & Copy number & Mouse & $\begin{array}{l}\text { RaggedEx } \\
\text { periment }\end{array}$ & 1.0 .0 & 2366 & 112 \\
\hline
\end{tabular}

Table 8: G\&T-seq dataset description, with assay types, molecular modes, number of specimens, number of features and number of cells

\section{Integrative analysis across modalities using data from SingleCellMultiModal}

Existing methods of integrative analysis of single-cell multimodal data have been recently reviewed [20]. Very briefly, some of the most popular current implementations are 1) the Seurat V4 R package which aims at vertical integration across several modal data types [56], 2) mixOmics [13] provides an extensive framework for data integration at molecular (P-integration, MINT [57]) and sample levels (N-integration, DIABLO [58]), and 3) Multi-Omics Factor Analysis, MOFA+ [14], a generalisation of Principal Components Analysis for inferring low-dimensional representation of multimodal data. Datasets provided by SingleCellMultiModal can be readily reshaped as input to any of these packages. We provide novel examples of such integrative analysis for exploratory visualization using SingleCellMultiModal datasets, produced within package documentation: MOFA+[14] on the 10X Multiome dataset (Figure 4). For more information, see Data Integration Methods. In addition, we provide a sample analysis on the SCOPE2 dataset, which can be found in SingleCellMultimodal's package vignette. 
bioRxiv preprint doi: https://doi.org/10.1101/2021.1027.466079. this version posted October 28, 2021. The copyright holder for this preprint (which was not certified by peer review) is the author/funder, who has granted bioRxiv a license to display the preprint in perpetuity. It is made From 10X Genomics

\section{A}

Raw seq data: FASTQ and sparseMatrix available

- RNA-seq

- ATAC-seq

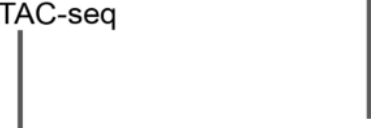

Pre-processing from SCMM
1. Data import in alternative formats:

- dgCMatrix (MTX)

- HDF5 on-disk

2. Integration of RNA-seq, ATAC-seq, metadata using MultiAssayExperiment

3. Automatic caching via ExperimentHub

\section{In SСMM package}

\section{B Call:}

scMultiome ("pbmc_10x")

and provided two MAE objects

A MultiAssayexperiment object of 2 listed

experiments with user-defined names and respective classes.

containing an Experimentlist class object of length 2:

[1] atac: Singlecellexperiment with 108344 rows and 10032 columns

[2] rna: singlecellexperiment with 36549 rows and 10032 columns

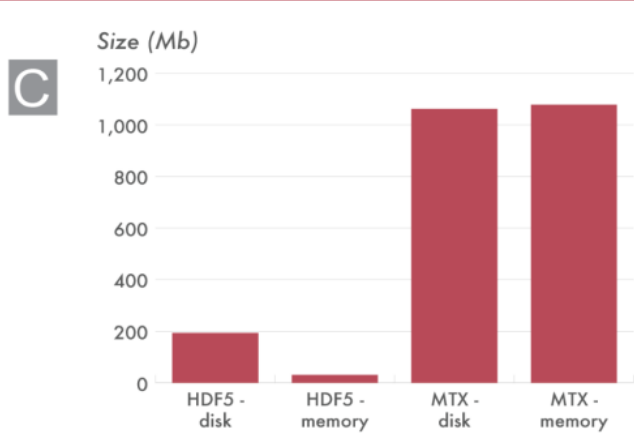

\section{MOFA+ analysis in BioConductor}

D
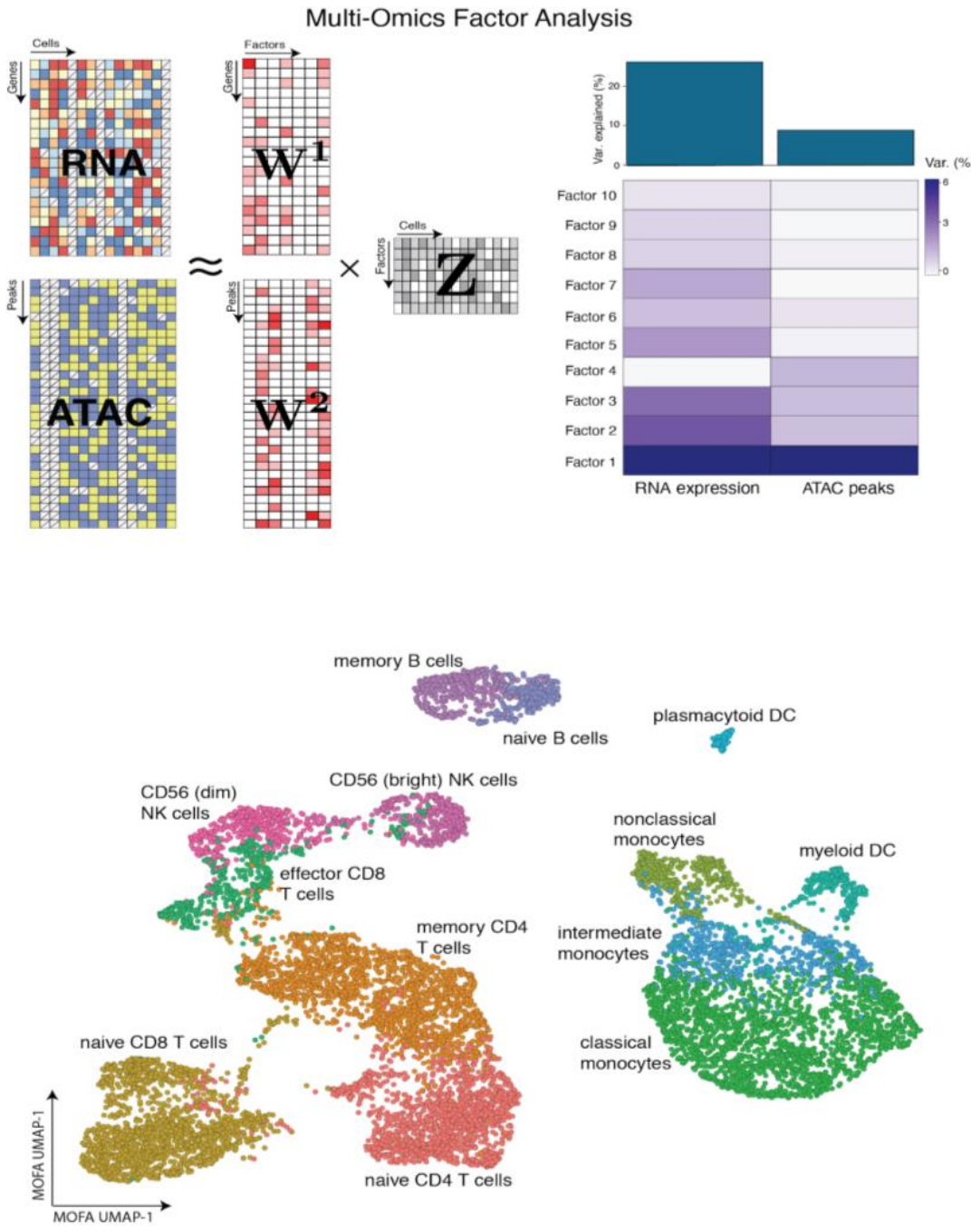
Figure 4: Summary of example integration using the 10X Genomic Multiome data from the SingleCellMultiModal package. (A): (left) from the 10X Genomic Multiome data resource, a sparse matrix and FASTA datasets provided, (right) pre-processing steps of source date required to work with of Multiome dataset provided in SingleCellMultiModal (C):

MultiAssayExperiment (MAE) objects returned when called (D), (E) data integration of 10x Genomic Multiome dataset, combining the chromatin accessibility data with the transcriptome data using MOFA+. (D): RNA-seq and ATAC-seq matrices used for weight factored analysis, (E): UMAP cluster of cell types based on factor analysis. For more detail on the analysis, see the Methods and the SingleCellMultiModal package vignette. Other datasets can be represented similarly: raw data processing and integration of data modes occur upstream of the SingleCellMultiModal package, users invoke a single command that creates a MultiAssayExperiment integrating appropriate memory-efficient objects, which are applied directly to downstream R/Bioconductor analyses.

\section{Methods}

\section{SingleCellMultiModal data package}

All datasets are distributed through the SingleCellMultiModal experimental data package in Bioconductor. This package employs ExperimentHub [59] for robust Cloud-based data download from AWS S3 buckets, with automatic local caching to avoid repetitive downloads. These methods are described in detail elsewhere for application to The Cancer Genome Atlas and cBioPortal [60]. Briefly, metadata and individual omics datasets are stored in ExperimentHub as simple core Bioconductor objects such as matrix, SparseMatrix, SingleCellExperiment, and RaggedExperiment. A simple user-facing convenience function is provided for each dataset that retrieves all necessary individual components, assembles a MultiAssayExperiment object [10], and returns this to the user. For very large matrices we employ HDF5 on-disk representation. Methods for users to access these datasets are documented in the SingleCellMultiModal package vignette and functions manual.

\section{CITE-Seq and ECCITE-Seq dataset}

The CITE-Seq contains two modalities of cord blood mononuclear cells, the transcripts (scRNA) and the cell surface proteins (SCADT) measured and preprocessed as described in the CITE-Seq landmark paper [3]. The PBMC UMI counts were retrieved from the GEO repository with accession number GSE100866 and then loaded in R to be transformed in matrix format and 
then be loaded as separate assays of a MultiAssayExperiment object. This latter object can be

retrieved from the SingleCellMultiModal package with the function call: CITEseq (DataType="cord_blood", dry.run=FALSE) .

The ECCITE-Seq has three modalities of the of peripheral blood mononuclear cells, (scRNA), the cell surface proteins ( $\mathrm{SCADT}$ ) and the Hashtagged Oligo ( $\mathrm{sCHTO}$ ) measured and preprocessed as described in the ECCITE-Seq landmark paper [4].

The PBMC modalities for the cutaneous T-cell lymphoma (CTCL) and controls (CTRL) were retrieved in TXT format from the GEO repository with accession number GSE126310 and then loaded in $\mathrm{R}$ to be transformed in matrix and data. frame format and then be loaded as separate assays of a MultiAssayExperiment object. The CRISPR perturbed scRNAs data are stored as data. frame in the object metadata to keep their original long format. This latter object can be retrieved from the SingleCellMultiModal with the function call:

CITEseq (DataType="peripheral_blood", dry.run=FALSE).

\section{Visual Cortex seqFISH dataset}

The seqFISH dataset has two different modalities, the spatial transcriptomics (seqFISH) and the single-cell RNA-seq, in two different versions. The main difference between the two versions are in the Single-cell RNA-seq counts data which in version 1.0.0 are provided as downloaded in CSV format from the GEO repository with accession number GSE71585, while the version 2.0.0 is a processed dataset [46] where only the genes with correspondence in the seqFISH dataset have been preserved. Methods of pre-processing are described at https://github.com/BIRSBiointegration/Hackathon. In both versions the seqFISH dataset is the processed version [46] as downloaded from https://cloudstor.aarnet.edu.au/plus/s/ZuBIXuzuvc9JMj3. Processed version of the seqFISH data were downloaded as TXT format for the coordinates (fcortex.coordinates.txt), as TSV format for the cell annotated labels (seqfish_labels.tsv) and TXT format for the counts 
(seqfish_cortex_b2_testing.txt). The data constitute a SpatialExperiment object with the counts as assay, the cell labels as colData and the coordinates stored as spatialData.

In the same way, the processed Single-cell RNA-seq data were downloaded as TXT format for the counts (tasic_training_b2.txt), as TSV format for the cell annotated labels (tasic_labels.tsv) to build a SingleCellExperiment object with the counts as assay and the cell labels as colData. Finally, the SingleCellExperiment and the SpatialExperiment have been loaded into a MultiAssayExperiment object as two different assays. The MultiAssayExperiment object can be retrieved with the function call, for example:

seqFISH (DataType="visual_cortex", dry.run=FALSE, version="2.๑.๑")

\section{Mouse Gastrulation scNMT dataset}

Preprocessing methods are described in full by Argelaguet et al. [22]. Briefly, RNA-seq libraries were aligned to the GRCm38 mouse genome build using HiSat235 (v.2.1.0). Gene expression counts were quantified from the mapped reads using featureCounts [61] with the Ensembl 87 gene annotation [62]. The read counts were log-transformed and size-factor adjusted using scran normalisation [63]. Bisulfite-seq libraries were aligned to the bisulfite converted GRCm38 mouse genome using Bismark [64]. Endogenous CpG methylation was quantified over ACG and TCG trinucleotides and GpC chromatin accessibility over GCA, GCC and GCT trinucleotides. Note that for GCG trinucleotides it is not possible to distinguish endogenous CpG methylation from induced GpC methylation. In addition, CGC positions were discarded because of off-target effects of the $\mathrm{GpC}$ methyltransferase enzyme [65].

For each $\mathrm{CpG}$ site in each cell we obtained binary methylation calls and for each GpC site in each cell we obtained binary accessibility calls. Notice that binary readouts is an exclusive property of single-cell bisulfite sequencing data, as for the vast majority of sites only one allele is observed per cell. This contrasts with bulk bisulfite sequencing data, where each dinucleotide typically contains multiple reads originating from different cells. 
Finally, we quantified DNA methylation and chromatin accessibility over genomic features by assuming a binomial model is assumed for each cell and feature, where the number of successes is the number of methylated $\mathrm{CpGs}$ (or GpCs) and the number of trials is the total number of CpGs (or GpCs) that are observed within the specific cell and genomic feature. Here, We quantified DNA methylation and chromatin accessibility rates over CpG islands, gene promoters, gene bodies and DNAse hypersensitive sites. All these data modalities were compiled together with the RNA expression into a MultiAssayExperiment object. The dataset can be loaded from within the SingleCellMultiModal package by the function call scNMT ("mouse_gastrulation", dry.run = FALSE). Code with the data processing pipeline is available in https://github.com/rargelaguet/scnmt gastrulation.

\section{X Multiome dataset}

PBMCs were extracted from a healthy donor after removing granulocytes through cell sorting.The dataset was downloaded as a CellRanger ARC output from

\section{https://support.10xgenomics.com/single-cell-multiome-atac-}

gex/datasets/1.0.0/pbmc granulocyte sorted 10k, which includes the gene expression matrix and the chromatin accessibility matrix quantified over ATAC peaks. The dataset included 11,909 cells with a median of 13,486 high-quality ATAC fragments per cell and a median of 1,826 genes expressed per cell. Data processing details, including the peak calling algorithm, can be found in https://support.10xgenomics.com/single-cell-multiome-atacgex/software/pipelines/latest/what-is-cell-ranger-arc. The dataset is provided as a MultiAssayExperiment [10] consisting of two SingleCellExperiment [9], one containing the single-cell RNA-seq read counts, and the other containing the binary ATAC peak matrix. The dataset can be loaded from within the SingleCellMultiModal package by the function call scMultiome ("pbmc_10x", dry.run = FALSE). 


\section{Macrophage differentiation SCOPE2 dataset}

The macrophage differentiation project contains two datasets: single-cell RNA-seq data and MS-SCP data. Upstream processing is described in detail in the SCoPE2 landmark paper [8]. Briefly, for the Single-cell RNA-seq dataset, the authors used CellRanger to align the reads and to build the UMI count matrices. Based on cell QC and manual inspection, they discarded cells containing less than $10^{4} \mathrm{UMI}$ barcodes. The resulting tables for two technical replicates were deposited in a GEO repository with accession GSE142392. For the MS-SCP dataset, the authors followed the workflow described in Figure 3, with identification and quantification steps performed using the MaxQuant software and additional protein quantification using a custom $\mathrm{R}$ script available on GitHub (https://github.com/SlavovLab/SCoPE2).

We retrieved the single-cell RNA-seq dataset from the GSE142392 repository. The MSSCP data and annotations were retrieved from CSV files available at the authors' website (https://scope2.slavovlab.net/docs/data). We formatted the Single-cell RNA-seq and the MSSCP data as two separate SingleCellExperiment objects without further processing. Because the Single-cell RNA-seq data is relatively large, it is stored as a sparse matrix using the HDF5 data format. We combined the two data objects in a single MultiAssayExperiment object. This latter object can be queried from the SingleCellMultimodal package with the function call SCOPE2("macrophage_differentiation", dry.run = FALSE).

\section{G\&T-seq dataset}

Raw sequencing data was obtained from the European Nucleotide Archive (ENA [66], accession PRJEB9051). The data was downloaded in fastq files for whole-genome and wholetranscriptome paired-end sequencing data for 112 mouse embryo cells. The data was processed as described in the step-by-step protocol of Macaulay et al. [49]. Preprocessing and mapping of genome sequencing data was carried out following steps $78-84$ of the protocol of Macaulay et al. [49], using Rsubread [67] for read trimming, alignment to the mm10 mouse 
reference genome, and removal of PCR-duplicate reads. DNA copy-number profiling was carried out following steps $85-87$, using bedtools [68] to convert BAM to BED files, and subsequently applying Ginkgo [69] for copy number determination. Preprocessing and mapping of transcriptome sequencing data was carried out following steps 94-96, using Rsubread [67] for read trimming and alignment to the $\mathrm{mm} 10$ mouse reference genome. Read counts for each gene were obtained using the featureCounts [61] function of the Rsubread package. The dataset is provided as a MultiAssayExperiment [10] consisting of (i) a SingleCellExperiment [9] storing the single-cell RNA-seq read counts, and (ii) a RaggedExperiment [54] storing integer copy numbers as previously described [55]. The dataset can be loaded from within the SingleCellMultiModal package by the function call GTseq $($ dry $\cdot$ run $=$ FALSE $)$.

\section{Data integration of the 10x Multiome data set}

For the integration of the 10x Multiome dataset we used MOFA+ [14] to obtain a latent embedding with contributom from both data modalities. The RNA expression was normalised using scran [63], followed by feature selection of the top 2000 most variable genes. The chromatin accessibility was normalised using TFIDF, followed by feature selection of the top 10,000 peaks with the highest mean accessibility. The MOFA model was trained with $K=15$ factors using default options. To obtain a non-linear embedding we applied UMAP [70] on the MOFA factors.

\section{Discussion}

Experimental data packages providing landmark datasets have historically played an important role in the development of new statistical methods in Bioconductor, from the classic acute lymphocytic leukemia $(A L L)$ microarray dataset [71] to the HSMMSingleCell single-cell RNA-seq 
dataset [72], as well as packages providing more extensive curated selections of standardized datasets in a specific realm [73]. Such packages greatly lower the barrier of access to relevant data for developers of scientific software, and provide a common testing ground for development and benchmarking. We present the SingleCellMultiModal Bioconductor experimental data package, to distribute landmark single-cell multimodal datasets in preintegrated immediately usable forms, utilizing standard Bioconductor data structures. Multimodal datasets are serialized as a MultiAssayExperiment object by a single command, without requiring users to perform data wrangling to link multiple 'omics profiles or to manage cells with incomplete data. We provide curated landmark datasets for a selection of key single-cell multimodal assays that will serve as benchmarks for the development and assessment of appropriate analysis methods in R/Bioconductor. We provide a brief review of the assays provided for the purpose of providing essential background to developers of statistical and bioinformatic methods, a summary of the data contained in each dataset, and examples of minimal code needed to access each dataset in an R/Bioconductor session. Methods of statistical analysis are reviewed in a recent complimentary paper [21].

Single-cell RNA-seq analysis methods in Bioconductor are well developed and widely used [9], setting the stage for new development in single-cell multimodal data analysis that will be facilitated by the SingleCellMultiModal experimental data package. Areas of active research include integrative systems biology across data modes, spatial statistics on high-dimensional data, dimension reduction and clustering [13], cell identification, multimodal batch correction, and new data structures for representation and analysis of large and spatially resolved singlecell multimodal data. These areas of research and their software products will be facilitated and made more interoperable by the easily accessible and uniformly represented data provided by this work. 


\section{Abbreviations}

\begin{tabular}{|l|l|}
\hline Abbreviation & Definition \\
\hline 10X Multiome & 10x Chromium Single Cell Multiome ATAC + Gene Expression \\
\hline ADT & Antibody derived tag \\
\hline CITE-Seq & Cellular Indexing of Transcriptomes and Epitopes by sequencing \\
\hline ECCITE-Seq & Expanded CRISPR CITE-Seq \\
\hline G\&T-seq & Genome and Transcriptome sequencing \\
\hline HDF5 & Hierarchical data format V5 \\
\hline HTO & Hashtag oligonucleotide \\
\hline LC & liquid chromatography \\
\hline m/z & mass over charge \\
\hline MOFA+ & Multi-Omics Factor Analysis V2 \\
\hline MS & mass spectrometry \\
\hline MS/MS & tandem MS \\
\hline PSM & peptide to spectrum match \\
\hline scNMT & single-cell Nucleosome, Methylation, and Transcriptome sequencing \\
\hline SCoPE2 & Single Cell ProtEomics by Mass Spectrometry V2 \\
\hline Single-cell RNA-seq & Single-cell RNA sequencing \\
\hline seqFISH & sequential Fluorescence In Situ Hybridization \\
\hline TMT & tandem mass tag \\
\hline UMI & unique molecular identifier sequence \\
\hline
\end{tabular}




\section{Data Availability Statement}

The data reviewed and curated in this review are publicly available under the Artistic 2.0 license as the SingleCellMultiModal Bioconductor package

(https://doi.org/doi:10.18129/B9.bioc.SingleCellMultiModal), with open development and issue tracking on Github (https://github.com/waldronlab/SingleCellMultiModal). The original 10X Genomics Multiome data are available from https://support.10xgenomics.com/single-cell$\underline{\text { multiome-atac-gex/datasets }}$

\section{Ethics declarations}

\section{Ethics approval and consent to participate}

Not applicable to the current manuscript.

\section{Competing interests}

The authors declare that they have no competing interests.

\section{Funding}

D.Ris, VC, MM, LW were supported by the National Cancer Institute of the National Institutes of Health (2U24CA180996). This work was supported in part by CZF2019-002443 (D.Rig, D.Ris, $\mathrm{MM}$ ) from the Chan Zuckerberg Initiative DAF, an advised fund of Silicon Valley Community Foundation. CV was supported by a PhD fellowship from the Belgian National Fund for Scientific Research (FNRS).

\section{Author contributions}

KBE prepared the manuscript, coordinated contributions, contributed Figure $1 \mathrm{~A}$, and co-created Figure 4. D.Rig performed pre-processing and addition of CITE-Seq, ECCITE-Seq and seqFISH datasets to the SingleCellMultiModal package, wrote the corresponding manuscripts main sections and Methods and Results. Contributed to the manuscript table and created Figures 1B and 2. MR created the SingleCellMultiModal Bioconductor package, and coordinated contributions to it. CV performed pre-processing and addition of SCOPE2 dataset to the SingleCellMultiModal package, wrote the corresponding sections of Methods and Results, and created Figure 3 (SCoPE2 workflow). RA performed pre-processing and addition of scNMT-seq + Multiome dataset to the SingleCellMultiModal package, wrote the corresponding sections of Methods and Results, and co-created Figure 4. L.Ge performed pre-processing and addition of the G\&T seq dataset to the SingleCellMultiModal package, and wrote the corresponding sections of Methods and Results. AC provided manuscript writing and edits. L.Ga contributed and supervised the SCOPE2 data preparation. VC, MM, D.Ris, and LW supervised the project. All authors read and approved the final manuscript. 


\section{References}

1. Schier AF. Single-cell biology: beyond the sum of its parts. Nat. Methods 2020; 17:17-20

2. . Method of the Year 2019: Single-cell multimodal omics. Nat. Methods 2020; 17:1

3. Stoeckius M, Hafemeister C, Stephenson W, et al. Simultaneous epitope and transcriptome measurement in single cells. Nat. Methods 2017; 14:865-868

4. Mimitou EP, Cheng A, Montalbano A, et al. Multiplexed detection of proteins, transcriptomes, clonotypes and CRISPR perturbations in single cells. Nat. Methods 2019; 16:409-412

5. Clark SJ, Argelaguet R, Kapourani CA, et al. ScNMT-seq enables joint profiling of chromatin accessibility DNA methylation and transcription in single cells e. Nat. Commun. 2018; 9:

6. Lubeck E, Coskun AF, Zhiyentayev T, et al. Single-cell in situ RNA profiling by sequential hybridization. Nat. Methods 2014; 11:360-361

7. Macaulay IC, Haerty W, Kumar P, et al. G\&T-seq: parallel sequencing of single-cell genomes and transcriptomes. Nat. Methods 2015; 12:519-522

8. Specht $\mathrm{H}$, Emmott E, Petelski AA, et al. Single-cell proteomic and transcriptomic analysis of macrophage heterogeneity using SCoPE2. Genome Biol. 2021; 22:50

9. Amezquita RA, Lun ATL, Becht E, et al. Orchestrating single-cell analysis with Bioconductor. Nat. Methods 2020; 17:137-145

10. Ramos M, Schiffer L, Re A, et al. Software for the Integration of Multiomics Experiments in Bioconductor. Cancer Res. 2017; 77:e39-e42

11. Huber W, Carey VJ, Gentleman R, et al. Orchestrating high-throughput genomic analysis with Bioconductor. Nat. Methods 2015; 12:115-121

12. Stuart T, Butler $A$, Hoffman $P$, et al. Comprehensive Integration of Single-Cell Data. Cell 2019; 177:1888-1902.e21

13. Rohart F, Gautier B, Singh A, et al. mixOmics: An R package for 'omics feature selection and multiple data integration. PLoS Comput. Biol. 2017; 13:e1005752

14. Argelaguet R, Arnol D, Bredikhin D, et al. MOFA+: a statistical framework for comprehensive integration of multi-modal single-cell data. Genome Biol. 2020;21:111

15. Kim HJ, Lin Y, Geddes TA, et al. CiteFuse enables multi-modal analysis of CITE-seq data. Bioinformatics 2020; 36:4137-4143

16. Wolf FA, Angerer P, Theis FJ. SCANPY: large-scale single-cell gene expression data analysis. Genome Biol. 2018; 19:15

17. Barkas N, Petukhov V, Nikolaeva D, et al. Joint analysis of heterogeneous single-cell RNAseq dataset collections. Nat. Methods 2019; 16:695-698

18. Gentleman R, Carey V, Huber W, et al. Bioinformatics and Computational Biology Solutions Using $R$ and Bioconductor. 2006;

19. Lawrence $\mathrm{M}$, Huber W, Pagès $\mathrm{H}$, et al. Software for computing and annotating genomic ranges. PLoS Comput. Biol. 2013; 9:e1003118

20. Lê Cao K-A, Abadi AJ, Davis-Marcisak EF, et al. Community-wide hackathons to identify central themes in single-cell multi-omics. Genome Biol. 2021;22:220

21. Al J Abadi Emily F. Davis-Marcisak Lauren Hsu Arshi Arora Alexis Coullomb Atul

Deshpande Yuzhou Feng Melanie Loth Vera Pancaldi Kris Sankaran Amrit Singh Joshua S.

Sodicoff Genevieve L. Stein-O'Brien Ayshwarya Subramanian Joshua D. Welch Yue You Ricard Argelaguet Vincent J. Carey Ruben Dries Casey S. Greene Susan Holmes Michael I. Love Matthew E. Ritchie Guo-Cheng Yuan Aedin C Culhane and Elana Fertig K-ALC. 'Communitywide hackathons establish foundations for emerging single cell data integration'. In submission 22. Argelaguet R, Clark SJ, Mohammed $\mathrm{H}$, et al. Multi-omics profiling of mouse gastrulation at single-cell resolution. Nature 2019; 576:487-491

23. Shi M, Annika K, Michael P. Nuclei Isolation from Tissue for 10x Multiome v1. protocols.io 
24. Tasic B, Menon V, Nguyen TN, et al. Adult mouse cortical cell taxonomy revealed by single cell transcriptomics. Nat. Neurosci. 2016; 19:335-346

25. Schepers K, Swart E, van Heijst JWJ, et al. Dissecting T cell lineage relationships by cellular barcoding. J. Exp. Med. 2008; 205:2309-2318

26. Conway JR, Lex A, Gehlenborg N. UpSetR: An R Package For The Visualization Of Intersecting Sets And Their Properties. bioRxiv 2017; 120600

27. Zhu Y, Piehowski PD, Zhao R, et al. Nanodroplet processing platform for deep and quantitative proteome profiling of 10-100 mammalian cells. Nat. Commun. 2018; 9:882

28. Kelly RT. Single-cell Proteomics: Progress and Prospects. Mol. Cell. Proteomics 2020; 19:1739-1748

29. Vanderaa C, Gatto L. Utilizing Scp for the analysis and replication of single-cell proteomics data. bioRxiv 2021; 2021.04.12.439408

30. Kelsey G, Stegle O, Reik W. Single-cell epigenomics: Recording the past and predicting the future. Science 2017; 358:69-75

31. Klemm SL, Shipony Z, Greenleaf WJ. Chromatin accessibility and the regulatory epigenome. Nat. Rev. Genet. 2019; 20:207-220

32. Smallwood SA, Lee HJ, Angermueller C, et al. Single-cell genome-wide bisulfite sequencing for assessing epigenetic heterogeneity. Nat. Methods 2014; 11:817-820

33. Angermueller $\mathrm{C}$, Clark SJ, Lee HJ, et al. Parallel single-cell sequencing links transcriptional and epigenetic heterogeneity. Nat. Methods 2016; 13:229-232

34. Song L, Crawford GE. DNase-seq: a high-resolution technique for mapping active gene regulatory elements across the genome from mammalian cells. Cold Spring Harb. Protoc. 2010; 2010:db.prot5384

35. Buenrostro JD, Wu B, Litzenburger UM, et al. Single-cell chromatin accessibility reveals principles of regulatory variation. Nature 2015; 523:486-490

36. Chen S, Lake BB, Zhang K. High-throughput sequencing of the transcriptome and chromatin accessibility in the same cell. Nat. Biotechnol. 2019; 37:1452-1457

37. Ma S, Zhang B, LaFave LM, et al. Chromatin Potential Identified by Shared Single-Cell

Profiling of RNA and Chromatin. Cell 2020; 183:1103-1116.e20

38. Pott S. Simultaneous measurement of chromatin accessibility, DNA methylation, and nucleosome phasing in single cells. Elife 2017; 6:

39. Chen KH, Boettiger AN, Moffitt JR, et al. RNA imaging. Spatially resolved, highly multiplexed RNA profiling in single cells. Science 2015; 348:aaa6090

40. Rodriques SG, Stickels RR, Goeva A, et al. Slide-seq: A scalable technology for measuring genome-wide expression at high spatial resolution. Science 2019; 363:1463-1467

41. Eng C-HL, Lawson M, Zhu Q, et al. Transcriptome-scale super-resolved imaging in tissues by RNA seqFISH. Nature 2019; 568:235-239

42. Righelli D, Weber LM, Crowell HL, et al. SpatialExperiment: infrastructure for spatially resolved transcriptomics data in R using Bioconductor. Cold Spring Harbor Laboratory 2021; 2021.01.27.428431

43. Moses L, Pachter L. Museum of Spatial Transcriptomics. bioRxiv 2021; 2021.05.11.443152 44. Shah S, Lubeck E, Zhou W, et al. In Situ Transcription Profiling of Single Cells Reveals Spatial Organization of Cells in the Mouse Hippocampus. Neuron 2016; 92:342-357

45. Zhu Q, Shah S, Dries R, et al. Identification of spatially associated subpopulations by combining scRNAseq and sequential fluorescence in situ hybridization data. Nat. Biotechnol. 2018;

46. birsauthors. White Paper for the Mathematical Frameworks for Integrative Analysis of Emerging Biological Data Types Workshop.

47. Taylor TH, Gitlin SA, Patrick JL, et al. The origin, mechanisms, incidence and clinical consequences of chromosomal mosaicism in humans. Hum. Reprod. Update 2014; 20:571-581 48. Campbell KR, Steif A, Laks E, et al. clonealign: statistical integration of independent single- 
cell RNA and DNA sequencing data from human cancers. Genome Biol. 2019; 20:54

49. Macaulay IC, Teng MJ, Haerty W, et al. Separation and parallel sequencing of the genomes and transcriptomes of single cells using G\&T-seq. Nat. Protoc. 2016; 11:2081-2103 50. Dey SS, Kester L, Spanjaard B, et al. Integrated genome and transcriptome sequencing of the same cell. Nat. Biotechnol. 2015; 33:285-289

51. Stuart T, Satija R. Integrative single-cell analysis. Nat. Rev. Genet. 2019; 20:257-272

52. Zahn $\mathrm{H}$, Steif A, Laks E, et al. Scalable whole-genome single-cell library preparation without preamplification. Nat. Methods 2017; 14:167-173

53. Zheng GXY, Terry JM, Belgrader $P$, et al. Massively parallel digital transcriptional profiling of single cells. Nat. Commun. 2017; 8:14049

54. Ramos M, Morgan M. RaggedExperiment: Representation of Sparse Experiments and Assays Across Samples.(2017). doi: 10.18129/B9. bioc. RaggedExperiment.

55. da Silva V, Ramos M, Groenen M, et al. CNVRanger: association analysis of CNVs with gene expression and quantitative phenotypes. Bioinformatics 2020; 36:972-973

56. Hao Y, Hao S, Andersen-Nissen E, et al. Integrated analysis of multimodal single-cell data. Cell 2021;

57. Rohart F, Eslami A, Matigian N, et al. MINT: a multivariate integrative method to identify reproducible molecular signatures across independent experiments and platforms. BMC Bioinformatics 2017; 18:128

58. Singh A, Shannon CP, Gautier B, et al. DIABLO: an integrative approach for identifying key molecular drivers from multi-omics assays. Bioinformatics 2019; 35:3055-3062

59. Pasolli E, Schiffer L, Manghi P, et al. Accessible, curated metagenomic data through

ExperimentHub. Nat. Methods 2017; 14:1023-1024

60. Ramos M, Geistlinger L, Oh S, et al. Multiomic Integration of Public Oncology Databases in Bioconductor. JCO Clin Cancer Inform 2020; 4:958-971

61. Liao Y, Smyth GK, Shi W. featureCounts: an efficient general purpose program for assigning sequence reads to genomic features. Bioinformatics 2014; 30:923-930

62. Yates A, Akanni W, Amode MR, et al. Ensembl 2016. Nucleic Acids Res. 2016; 44:D710-6

63. Lun ATL, McCarthy DJ, Marioni JC. A step-by-step workflow for low-level analysis of single-

cell RNA-seq data with Bioconductor. F1000Research 2016; 5:2122

64. Krueger F, Andrews SR. Bismark: a flexible aligner and methylation caller for Bisulfite-Seq applications. Bioinformatics 2011; 27:1571-1572

65. Kelly TK, Liu Y, Lay FD, et al. Genome-wide mapping of nucleosome positioning and DNA methylation within individual DNA molecules. Genome Res. 2012; 22:2497-2506

66. Harrison PW, Ahamed A, Aslam R, et al. The European Nucleotide Archive in 2020. Nucleic Acids Res. 2021; 49:D82-D85

67. Liao Y, Smyth GK, Shi W. The R package Rsubread is easier, faster, cheaper and better for alignment and quantification of RNA sequencing reads. Nucleic Acids Res. 2019; 47:e47

68. Quinlan AR. BEDTools: The Swiss-Army Tool for Genome Feature Analysis. Curr. Protoc.

Bioinformatics 2014; 47:11.12.1-34

69. Garvin T, Aboukhalil R, Kendall J, et al. Interactive analysis and assessment of single-cell copy-number variations. Nat. Methods 2015; 12:1058-1060

70. Mclnnes L, Healy J, Melville J. UMAP: Uniform Manifold Approximation and Projection for Dimension Reduction. arXiv [stat.ML] 2018;

71. Li X. ALL Bioconductor package. 2004;

72. Trapnell C. HSMMSingleCell: Single-cell RNA-Seq for differentiating human skeletal muscle myoblasts (HSMM). R package version 2014; 1140 :

73. Ganzfried BF, Riester M, Haibe-Kains B, et al. curatedOvarianData: clinically annotated data for the ovarian cancer transcriptome. Database 2013; 2013:bat013 